\title{
A Controllable Canonical Form for Constant-ratio Proportional and Derivative (CRPD) Feedbacks in Generalized Systems
}

\author{
Hung-Chou Chen and Fan-Ren Chang ${ }^{\dagger}$ \\ Department of Electrical Engineering \\ National Taiwan University \\ Taipei, Taiwan, 10764 \\ Republic of China
}

\begin{abstract}
This paper deals with the eigenvalue placement of controllable generalized systems of the form $\hat{E} \dot{x}(t)=\hat{A} x(t)+$ $\hat{B} u(t)$ by constant-ratio proportional and derivative (CRPD) feedback control of the form $u(t)=\hat{K}(\rho \dot{x}(t)-x(t))+w(t)$. The problem discussed is an extension of the traditional state feedback control law, which is the case with $\hat{E}=I$ and $\rho=0$. A controllable canonical form and its transformation matrices are used to solve our problem. Based on the developed canonical transformation, pole assignment of generalized systems can be implemented easily. The development of our method depends crucially on the properties of standard form generalized systems. One illustrative example is included.
\end{abstract}

Keywords- Generalized systems, Standard forms, Eigenvalue placement, Controllable forms, State feedback.

\section{Introduction}

Generalized systems (singular systems, descriptor systems, generalized state space systems, or semistate systems), have attracted much attention in recent years (Lewis, 1986). There are extensive applications of generalized systems in power systems, robotic manpulators, circuits, economic systems, and large-scale systems.

The problem of eigenvalue assignment via proportional and derivative feedback have been studied by Mukundan and Dayawansa (1983), Syrmos and Lewis (1991), etc.. Proportional and derivative feedback can modify the system structure by replacing the poles so as to decrease the suceptibility to noise and improve performance. Dealing with two feedback gain matrices always results in a complicated computation procedure. In Zhou (1984), and Shayman and Zhou (1987) a theoretical classification of generalized systems using constant-ratio proportional and derivative (CRPD) feedback was presented.

We consider the linear time-invariant generalized system of the form:

$$
\hat{\Sigma}: \hat{E} \dot{x}(t)=\hat{A} x(t)+\hat{B} u(t)
$$

$x(t) \in \Re^{n}$ is the state vector, $u(t) \in \Re^{r}$ is the input vector, $\hat{E}$, $\hat{A}$ and $\hat{B}$ are real constant matrices of compatible dimensions. The matrix $\hat{E}$ may be singular. $\{\hat{E}, \hat{A}\}$ is commonly called as the general form since no special patterns are assumed.

Before entering the main content, some basic concepts of generalized systems, such as regularity, controllability, and eigenvalues, shall be introduced first.

Definition 1: (Gantmacher, 1977)

If $\operatorname{det}(s \hat{E}-\hat{A}) \not \equiv 0$ then $(s \hat{E}-\hat{A})$ is a regular pencil. The generalized system $(1)$ is regular if its associated pencil $(s \hat{E}-\hat{A})$ is regular.

$\dagger$ To whom all correspondence should be addressed. 0-7803-0510-8/92\$03.00 @1992IEEE
Lemma 1: $(s \hat{A}-\hat{E})$ is regular if and only if $(s \hat{E}-\hat{A})$ is regular.

Proof:

1. Sufficient part:

Since $\operatorname{det}(s \hat{E}-\hat{A}) \not \equiv 0$, there exists at least a scalar $s_{1}$, s.t. $\operatorname{det}\left(s_{1} \hat{E}-\hat{A}\right) \neq 0$. There are two possibilities for $s_{1}$.

(a) If $s_{1} \neq 0$, then $\operatorname{det}\left(s_{1} \hat{E}-\hat{A}\right)=\left(-s_{1}\right)^{n} \operatorname{det}\left(\frac{-1}{s_{1}} \hat{A}-\right.$ $\hat{E}) \neq 0$, where $n$ is the size of matrices $\hat{E}$ and $\hat{A}$. Since $\left(-s_{1}\right)^{n} \neq 0$, we have $\operatorname{det}\left(\frac{-1}{s_{1}} \hat{A}-\hat{E}\right) \neq 0$. So, $\operatorname{det}(s \hat{A}-\hat{E}) \not \equiv 0$.

(b) If $s_{1}=0$, then $\operatorname{det}\left(s_{1} \hat{E}-\hat{A}\right)=\operatorname{det}(-\hat{A}) \neq 0$ and $\hat{A}$ is invertible. Assume $\hat{s}_{1} \notin$ eigenvalues of $\left.\hat{E} \hat{A}^{-1}\right\}$, then $\operatorname{det}\left(\hat{s}_{1} I-\hat{E} \hat{A}^{-1}\right) \neq 0$. Note that $\operatorname{det}\left(\hat{s}_{1} I-\hat{E} \hat{A}^{-1}\right)=$ $\operatorname{det}\left(\hat{s}_{1} \hat{A}-\hat{E}\right) \operatorname{det}\left(\hat{A}^{-1}\right) \neq 0$. Since $\hat{A}^{-1}$ is nonsingular, we have $\operatorname{det}\left(s_{1} \hat{A}-\hat{E}\right) \neq 0$. So, $\operatorname{det}(s \hat{A}-\hat{E}) \neq 0$.

2. Necessary part:

To verify the necessary condition can be obtained in a similiar way.

Definition 2: (Cobb, 1984)

If (i) $\left[\begin{array}{ll}s \hat{E}-\hat{A} & \hat{B}\end{array}\right]$ has full rank for all finite $s$ and (ii) $\left[\begin{array}{ll}\hat{E} & \hat{B}\end{array}\right]$ has full rank then the generalized system (1) is controllable. $\square$

Definition 3: (Lewis, 1986)

Let $(s \hat{E}-\hat{A})$ be a regular pencil. If there exists a finite scalar $\hat{\lambda}_{k} \operatorname{such}$ that $\operatorname{det}\left(\hat{\lambda}_{k} \hat{E}-\hat{A}\right)=0$, then $(s \hat{E}-\hat{A})$ has a finite eigenvalue $\hat{\lambda}_{k}$. If $\hat{E}$ is singular, then $(s \hat{E}-\hat{A})$ has an infinite eigenvalue. The finite and infinite eigenvalues of $(s \hat{E}-\hat{A})$ are the finite and infinite poles of the associated generalized system (1).

Zhou (1984) has investigated the eigenvalue assignment problem under constant-ratio proportional and derivative (CRPD) feedback from the theoretical viewpoint. A main result is as follows:

Lemma 2: (Zhou, 1984)

If the generalized system (1) is controllable, then the control law of CRPD feedback ( i.e. $u(t)=\hat{K}(\rho \dot{x}(t)-x(t))+w(t)$, where $\rho$ is a scalar ) can arbitrarily relocate eigenvalues ( subject to complex conjugate pairs ) and eliminate impulse behaviour simultaneously.

As this lemma is useful in theoretical analyses, the method for finding $\hat{K}$ and $\rho$ was not deeply explored. The purpose of this paper is to provide a controllable canonical form for the generalized system (1), such that the implementation of CRPD feedback becomes apparent. The development of our method depends crucially on the properties of standard form general- 
ized systems. We shall transform $\{\hat{E}, \hat{A}, \hat{B}\}$ into the CRPD controllable canonical form $\{\tilde{E}, \tilde{A}, \tilde{B}\}$. Under this arrangement, the gain matrix $\hat{K}$ and ratio $\rho$ of Lemma 2 , can be easily computed. In the following, we will describe formulation of the problem first.

\section{Problem formulation}

Assume the generalized system of (1) is regular and controllable. The purpose of this paper is to find the matrix $\hat{K}$ and $\rho$ in the CRPD state feedback control law,

$$
u(t)=\hat{K}(\rho \dot{x}(t)-x(t))+w(t)
$$

such that the closed-loop system,

$$
\hat{\Sigma}_{c}: \hat{E}_{\mathrm{c}} \dot{x}(t)=\hat{A}_{c} x(t)+\hat{B} w(t)
$$

where

$$
\hat{E}_{c} \triangleq \hat{E}-\rho \hat{B} \hat{K}, \quad \hat{A}_{c} \triangleq \hat{A}-\hat{B} \hat{K}
$$

satisfies the following requirements:

1. The closed-loop finite poles can be arbitrarily assigned to any places subject to complex conjugate pairs.

2. Regularity is guaranteed, i.e. $\left|s \hat{E}_{c}-\hat{A}_{c}\right| \not \equiv 0$.

If the number of finite poles of (3) equals to $\operatorname{rank}\left(\hat{E}_{c}\right)$, then the response caused by any initial conditions are impulse free.

In this paper, the generalized system's standard form and its basic properties discussed in Section II. CRPD controllable canonial form is introduced and poles assignment via the CRPD feedback is investigated in Section III. In Section IV, a numerical example to illustrate the proposed method is provided. Conclusions are stated in the last section.

\section{Properties of Standard Generalized Systems}

Definition 4: (Nikoukhan, 1987)

$(s \hat{E}-\hat{A})$ is a standard pencil if there exist some scalars $\alpha$ and $\beta$ s.t.

$$
\alpha \hat{E}+\beta \hat{A}=I
$$

The generalized system (1) is standard if its associated pencil is standard.

Note that $(s I-\hat{A})$ is a standard pencil $(\alpha=1, \beta=0)$. Also, every regular generalized system (1) can be transformed into a standard one. We just premultiply (1) by $(\hat{E}-\rho \hat{A})^{-1}$, where $\rho$ is any scalar s.t. $(\hat{E}-\rho \hat{A})$ is nonsingular, then the generalized system becomes

$$
\Sigma: E \dot{x}(t)=A x(t)+B u(t)
$$

where

$E=(\hat{E}-\rho \hat{A})^{-1} \hat{E}$

$A=(\hat{E}-\rho \hat{A})^{-1} \hat{A}$

$B=(\hat{E}-\rho \hat{A})^{-1} \hat{B}$

In doing so, we do not change the system or the 'state' variable $x$. The new system $\Sigma$ of (5) is now a standard one by taking $\alpha=1$ and $\beta=-\rho$ (i.e. $E-\rho A=I$ ). We will now investigate some important propertites of standard pencils.

Lemma 3:

Let $(s E-A)$ be a standard pencil, which satisfies $E-\rho A=$ $I$. The eigenvalue, $\lambda_{A}$, of $A$ and the finite eigenvalue, $\lambda_{R}$, of $(s E-A)$ are related by $\lambda_{R}=\frac{\lambda_{A}}{\rho \lambda_{A}+1}$ if $\rho \lambda_{A}+1 \neq 0$.
Proof:

If $\lambda_{A}$ is the eigenvalue of $A$, then $\operatorname{det}\left(\lambda_{A} I-A\right)=0$. Note that

$$
\begin{aligned}
\lambda_{A} I-A & =\lambda_{A}(E-\rho A)-A \\
& =\left(\rho \lambda_{A}+1\right)\left[\left(\frac{\lambda_{A}}{\rho \lambda_{A}+1}\right) E-A\right]
\end{aligned}
$$

Hence

$$
\operatorname{det}\left(\lambda_{A} I-A\right)=\left(\rho \lambda_{A}+1\right)^{n} \operatorname{det}\left[\left(\frac{\lambda_{A}}{\rho \lambda_{A}+1}\right) E-A\right]=0
$$

where $n$ is the size of matrices $E, A$. Since $\left(\rho \lambda_{A}+1\right) \neq 0$, it is clear $\frac{\lambda_{A}}{\rho \lambda_{A}+1}$ is the finite eigenvalue $\lambda_{R}$ of $(s E-A)$.

\section{Lemma 4:}

Let $(s E-A)$ be a standard pencil, which satisfies $E-\rho A=I$. If $A$ has an eigenvalue $\lambda_{A}$, s.t. $\rho \lambda_{A}+1=0$, then $(s E-A)$ has an infinite eigenvalue. If $(s E-A)$ has an infinite eigenvalue, then $A$ has at least one eigenvalue $\lambda_{A}$, s.t. $\rho \lambda_{A}+1=0$.

Proof:

1. If $\lambda_{A}$ is the eigenvalue of $A$ and $\rho \lambda_{A}+1=0$, then $\operatorname{det}\left(\lambda_{A} I-\right.$ A) $=0$. Note that

$$
\lambda_{A} I-A=\lambda_{A}(E-\rho A)-A=\lambda_{A} E
$$

Hence,

$$
\operatorname{det}\left(\lambda_{A} I-A\right)=\lambda_{A}^{n} \operatorname{det}(E)=0
$$

where $n$ is the size of matrices $E, A$. Since $\rho \lambda_{A}+1=0$, it implies $\lambda_{A} \neq 0$. From (7), it is clear that $\operatorname{det}(E)=0$. By Definition 3, $E$ matrix being singular means $(s E-A)$ has an infinite eigenvalue.

2. If $(s E-A)$ has an infinite eigenvalue, then $E$ is singular. Let $M$ be the model matrix of $A$, Then $M^{-1} A M=$ block$\operatorname{diag}\left\{\Lambda_{i}\right\}$, where

$$
\Lambda_{i}=\left[\begin{array}{cccc}
\lambda_{i} & 1 & & \\
& \ddots & \ddots & \\
& & \ddots & 1 \\
& & & \lambda_{i}
\end{array}\right]
$$

and $\lambda_{i}$ is the eigenvalue of $A$. From $E=\rho A+I$, then $M^{-1} E M$ becomes block-diag $\left\{D_{i}\right\}$, where

$$
D_{i}=\left[\begin{array}{cccc}
\lambda_{i} \rho+1 & 1 & & \\
& \ddots & \ddots & \\
& & \ddots & 1 \\
& & & \lambda_{i} \rho+1
\end{array}\right] .
$$

Since $E$ is singular, there exists at least one $\lambda_{i}$, s.t. $\lambda_{i} \rho+$ $1=0$.

From Lemma 4, we can count the number of infinite eigenvalues of $(s E-A)$ by counting the number of eigenvalues of $A$, $\lambda_{A}$, which satisfying $\rho \lambda_{A}+1=0$. By Lemma 3 and 4, the eigenvalues of $(s E-A), \lambda_{R}$, and those of $A, \lambda_{A}$, have a one-to-one mapping.

As we have seen in (6), a regular generalized system $\hat{\Sigma}$ of (1) can be easily transformed into a standard one $\Sigma$ of (5). 
Lemma 5 shows that both systems share the same eigenvalues and controllability conditions.

\section{Lemma 5:}

1. Systems $\hat{\Sigma}$ of (1) and $\Sigma$ of (5) have the same poles.

2. $\hat{\Sigma}$ of (1) is controllable if and only if $\Sigma$ of (1) is controllable.

\section{Proof:}

1. From (6.a) and (6.b), $(s E-A)=(\hat{E}-\rho \hat{A})^{-1}(s \hat{E}-\hat{A})$. So, (1) and (5) share the same finite poles. Also, singularity of $\hat{E}$ and $E=(\hat{E}-\rho \hat{A})^{-1} \hat{E}$ is the same. Hence, (1) and (5) share the same infinite poles.

2. $\operatorname{rank}[s E-A \quad B]=\operatorname{rank}(\hat{E}-\rho \hat{A})^{-1}\left[\begin{array}{l}s \hat{E}-\hat{A} \quad \hat{B}\end{array}\right]=\operatorname{rank}(s \hat{E}-$ $\left.\begin{array}{ll}\hat{A} & \hat{B}\end{array}\right]$, and $\operatorname{rank}\left[\begin{array}{ll}E & B\end{array}\right]=\operatorname{rank}(\hat{E}-\rho \hat{A})^{-1}\left[\begin{array}{ll}\hat{E} & \hat{B}\end{array}\right]=$ $\operatorname{rank}[\hat{E} \quad \hat{B}]$.

If the CRPD control low (2), $u(t)=\hat{K}(\rho \dot{x}(t)-x(t))+w(t)$, in which $\rho$ is the same one as that of (6), is applied to (1) and (5), then the closed-loop systems become (8) and (9) respectively.

$\hat{\Sigma}_{c}: \hat{E}_{c} \dot{x}(t)=\hat{A}_{c} x(t)+\hat{B} w(t)$

where $\hat{E}_{c} \triangleq \hat{E}-\rho \hat{B} \hat{K}, \quad \hat{A}_{c} \triangleq \hat{A}-\hat{B} \hat{K}$

$\Sigma_{c}: E_{c} \dot{x}(t)=A_{c} x(t)+B w(t)$

where $E_{\mathrm{c}} \triangleq E-\rho B \hat{K}, \quad A_{c} \triangleq A-B \hat{K}$

Lemma 6:

1. The closed-loop system $\hat{\Sigma}_{c}$ of (8) is regular.

2. The closed-loop system $\Sigma_{c}$ of (9) is standard.

\section{Proof:}

1. From (8.b), $\hat{E}_{c}-\rho \hat{A}_{c}=\hat{E}-\rho \hat{A}$. Since $\rho$ is selected s.t. ( $\hat{E}-\rho \hat{A})$ being nonsingular, (8.a) is a regular generalized system by Lemma 1 .

2. From (9.b), $E_{c}-\rho A_{c}=E-\rho A=I$ by (6). Hence, (9.a) is a standard generalized system.

Lemma 7:

$\hat{\Sigma}_{c}$ of $(8)$ and $\Sigma_{c}$ of $(9)$ have the same set of poles.

Proof:

$$
\begin{aligned}
\left(s E_{c}-A_{c}\right) & =s(E-\rho B \hat{K})-(A-B \hat{K}) \\
& =(\hat{E}-\rho \hat{A})^{-1}[s(\hat{E}-\rho \hat{B} \hat{K})-(\hat{A}-\hat{B} \hat{K})] \\
& =(\hat{E}-\rho \hat{A})^{-1}\left[s \hat{E}_{c}-\hat{A}_{c}\right]
\end{aligned}
$$

Hence, (8) and (9) have the same set of finite poles, which are roots of $\operatorname{det}\left(s \hat{E}_{c}-\hat{A}_{c}\right)=0$. Also, singularity of $\hat{E}_{c}$ and $E_{c}=(\hat{E}-\rho \hat{A})^{-1} \hat{E}_{c}$ is the same. So (8) and (9) have the same infinite poles.

\section{Theorem 1} (9).

Lets consider the closed-loop systems $\Sigma_{c}$ of $(8)$ and $\Sigma_{c}$ of

1. If $\rho$ and $\hat{K}$ are selected s.t. $\rho \lambda_{A_{c i}}+1 \neq 0$, where $\lambda_{A_{c i}}$ is an eigenvalue of $A_{c}=A-B \hat{K}$, then $\lambda_{R_{o i}}=\frac{\lambda_{A_{c i}}}{\rho \lambda_{A_{c i}}+1}$ is a finite pole of (8)

2. If $\rho$ and $\hat{K}$ are selected s.t. $\rho \lambda_{A_{c i}}+1=0$, where $\lambda_{A_{c i}}$ is an eigenvalue of $A_{c}=A-B \hat{K}$, then (8) has infinite poles.
1. From Lemma 6 part $2,(9)$ is a standand generalized system. From Lemma 3, $\lambda_{R_{c i}}=\frac{\lambda_{A_{c i}}}{\rho \lambda_{A}+1}$ is a finite pole of (9). Also, Lemma 7 has shown that $(8)$ and (9) have the same set of eigenvalues, so $\lambda_{R_{c i}}$ is a finite pole of (8).

2. From Lemma 6 par 2, Lemma 4 and Lemma 7, (8) has infinite poles.

As a summary of this section, we have shown that the poles of the closed-loop system $\Sigma_{c}$ of (8) are determined by the eigenvalue of $A_{c}, \lambda_{A_{i c}}$. For the design consideration, an effective algorithm to assign $A_{c}$ 's eigenvalues is an important issue.

\section{CRPD Controllable Canonical Forms and}

\section{Pole Assignment}

Theorem 2:

$\hat{\Sigma}$ of $(1)$ is controllable, if and only if $C=\left[\begin{array}{lllll}B & A B & A^{2} B & \cdots & A^{n-1} B\end{array}\right]$ has full rank.

Proof:

$C$ has full rank if and only if $\operatorname{rank}\left[\lambda_{A} I-A \quad B\right]=n$ for all eigenvalues, $\lambda_{A}$, of $A$. We consider two conditions.

1. $\rho \lambda_{A}+1 \neq 0$

$$
\begin{aligned}
& \operatorname{rank}\left[\lambda_{A} I-A \quad B\right] \\
& =\operatorname{rank}\left[\lambda_{A}(E-\rho A)-A \quad B\right] \\
& =\operatorname{rank}\left[\lambda_{A} E-\left(\rho \lambda_{A}+1\right) A \quad B\right] \\
& =\operatorname{rank}\left[\frac{\lambda_{A}}{\left(\rho \lambda_{A}+1\right)} E-A \quad B\right] \\
& =\operatorname{rank}\left[\lambda_{R} E-A \quad B\right] \\
& =n
\end{aligned}
$$

2. $\rho \lambda_{A}+1=0$

$$
\begin{aligned}
\operatorname{rank} & {\left[\begin{array}{ll}
\lambda_{A} I-A & B
\end{array}\right] } \\
& =\operatorname{rank}\left[\begin{array}{ll}
\lambda_{A} E-\left(\rho \lambda_{A}+1\right) A & B
\end{array}\right] \\
& =\operatorname{rank}\left[\begin{array}{ll}
\lambda_{A} E & B
\end{array}\right] \\
& =\operatorname{rank}\left[\begin{array}{ll}
E & B
\end{array}\right] \\
& =n
\end{aligned}
$$

From 1 and $2, \Sigma$ of $(5)$ is controllable. By Lemma $5(2), \hat{\Sigma}$ of (1) is controllable. Thus we have complete the sufficient part of this theorem. The necessary part can be proved in a similar way.

In the following, we shall transform $\{A, B\}$ into the controllable canonical form discussed in Chen (1980, pp345 ). It is assumed that the controllability indices are $\kappa_{1}, \kappa_{2}, \ldots, \kappa_{r}$, let $n_{0}=0$ and $n_{i}=\sum_{i=0}^{i} \kappa_{i}, i=1,2,3, \ldots, r$, so $n_{r}=n$. Define the matrix

$$
M \triangleq\left[b_{1} A b_{1} \cdots A^{\kappa_{1}-1} b_{1} \vdots b_{2} A b_{2} \cdots A^{\kappa_{2}-1} b_{2} \vdots \cdots\right.
$$

$b_{r} A b_{r} \cdots A^{\alpha_{r}-1} b_{r}$

then $M$ is nonsingular. We compute $M^{-1}$ and denote its rows by $e_{i j}, i=1,2, \ldots, r, j=1,2, \ldots, \kappa_{i}$. Now we use $e_{i \kappa_{i}}, i=$ $1,2, \ldots, r$ to form the square matrix $T$,

Proof: 


$$
M^{-1} \triangleq\left[\begin{array}{c}
e_{11} \\
e_{12} \\
\vdots \\
e_{1 \kappa_{1}} \\
\cdots \\
e_{21} \\
e_{22} \\
\vdots \\
e_{2 \kappa_{2}} \\
\cdots \\
\vdots \\
\cdots \\
e_{r 1} \\
e_{r 2} \\
\vdots \\
e_{r \kappa_{r}}
\end{array}\right], \quad T \triangleq\left[\begin{array}{c}
e_{1 \kappa_{1}} \\
e_{1 \kappa_{1}} A \\
\vdots \\
e_{1 \kappa_{1}} A^{\kappa_{1}-1} \\
\cdots \\
e_{2 \kappa_{2}} \\
e_{2 \kappa_{2}} A \\
\vdots \\
e_{2 \kappa_{2}} A^{\kappa_{2}-1} \\
\cdots \\
\vdots \\
\cdots \\
e_{r \kappa_{r}} \\
e_{r \kappa_{r}} A \\
\vdots \\
e_{r \kappa_{r}} A^{\kappa_{r}-1}
\end{array}\right]
$$

then $T$ is nonsingular. The controllable canonical form $\{\tilde{A}, \tilde{B}\}$ are given by similarity transformations

$$
\tilde{A}=T A T^{-1}=\left[\tilde{A}_{i j}\right] \text { for } i=1, \ldots, r \text { and } j=1, \ldots, r
$$
where

$$
\begin{aligned}
\tilde{A}_{i i} & =\left[\begin{array}{ccccc}
0 & 1 & 0 & & 0 \\
0 & 0 & 1 & & 0 \\
& & & \ddots & \\
a_{n_{i}\left(n_{(i-1)}+1\right)} & \cdots & \cdots & \cdots & a_{n_{i} n_{i}}
\end{array}\right]_{\kappa_{i} \times \kappa_{i}} \\
\tilde{A}_{i j} & =\left[\begin{array}{ccccc}
0 & 0 & 0 & & 0 \\
0 & 0 & 0 & & 0 \\
& & & \ddots & \\
a_{n_{i}\left(n_{(j-1)}+1\right)} & \cdots & \cdots & \cdots & a_{n_{i} n_{j}}
\end{array}\right]_{\kappa_{i} \times \kappa_{j}} \text { for } i \neq j
\end{aligned}
$$

and

$$
\tilde{B}=T B=\left[\begin{array}{c}
\tilde{B}_{1} \\
\vdots \\
\tilde{B}_{i} \\
\vdots \\
\tilde{B}_{\mathrm{r}}
\end{array}\right], \text { for } i=1, \ldots, r
$$

where

$$
\tilde{B}_{i}=\left[\begin{array}{ccccc}
0 & & & & 0 \\
0 & \ldots & & \ldots & 0 \\
\vdots & \ldots & & \ldots & \vdots \\
0 & 0 & b_{n_{i} i}=1 & b_{n_{i}(i+\cdots)} & b_{n_{i} r}
\end{array}\right]_{\kappa_{i} \times r}
$$

By the same transformation, we have

$$
\begin{aligned}
& \tilde{E}=T E T^{-1}=T(I+\rho A) T^{-1}=\left[\tilde{E}_{i j}\right] \\
& \text { for } i=1, \ldots, r \text { and } j=1, \ldots, r \\
& \begin{array}{l}
\tilde{E}_{i i}=\left[\begin{array}{ccccc}
1 & \rho & 0 & & 0 \\
0 & 1 & \rho & & 0 \\
& & & \ddots & \\
\rho a_{n_{i}\left(n_{(i-1)}+1\right)} & \cdots & \cdots & \cdots & 1+\rho a_{n_{i} n_{i}}
\end{array}\right]_{\kappa_{i} \times \kappa_{i}} \\
\tilde{E}_{i j}=\left[\begin{array}{ccccc}
0 & 0 & 0 & & 0 \\
0 & 0 & 0 & & 0 \\
& & & \ddots & \\
\rho a_{n_{i}\left(n_{(j-1)}+1\right)} & \cdots & \cdots & \cdots & \rho a_{n_{i} n_{j}}
\end{array}\right]_{\kappa_{i} \times \kappa_{j}} \text { for } i \neq j
\end{array}
\end{aligned}
$$

where the unfilled positions are all zeros.
Definition 5:

The triple $\{\tilde{E}, \tilde{A}, \tilde{B}\}$ is a CRPD controllable canonical form.

Given the CRPD controllable canonical form

$$
\tilde{\Sigma}: \tilde{E} \dot{\tilde{x}}(t)=\tilde{A} \tilde{x}(t)+\tilde{B} w(t)
$$

where $\tilde{x}(t)=T x(t)$, there exists typical procedures to find $\tilde{K}$, s.t. $\tilde{A}_{c} \triangleq \tilde{A}-\tilde{B} \tilde{K}$ has the desired eigenvalues. The closed-loop of CRPD control law $u(t)=\tilde{K}(\rho \dot{\tilde{x}}(t)-\tilde{x}(t))+w(t)$ becomes

$\tilde{\Sigma}_{c}: \tilde{E}_{c} \dot{\tilde{x}}(t)=\tilde{A}_{c} \tilde{x}(t)+\tilde{B} w(t)$

where $\tilde{E}_{c} \triangleq \tilde{E}-\rho \tilde{B} \tilde{K}, \quad \tilde{A}_{c} \triangleq \tilde{A}-\tilde{B} \tilde{K}$ Compare $\tilde{\Sigma}_{c}$ of (13), $\Sigma_{c}$ of (9) and $\hat{\Sigma}_{c}$ of (8), we find that they have the same poles by Lemma 7 .

For a given controllable generalized system $\hat{\Sigma}$ of (1), the CRPD feedback design procedure for pole placement is summarized as follows:

1. Select $\rho$, which satisfying $\operatorname{det}(\hat{E}-\rho \hat{A}) \neq 0 . \hat{\Sigma}$ of $(1)$ can be transformed into the standard system, $\Sigma$ of (5) by (6).

2. Similarily transform $\Sigma$ of (5) into the CRPD controllable canonical form $\tilde{\Sigma}$ of (12) by the matrix $T$ of $(10 . c)$.

3. Find $\tilde{K}$ so that $\tilde{A}_{c} \triangleq \tilde{A}-\tilde{B} \tilde{K}$ has the desired eigenvalues $\lambda_{\bar{A}_{c i}}$, which are associated to the closed-loop finite poles $\lambda_{R_{c i}}=\frac{\lambda_{\hat{A}_{c i}}}{\rho \lambda_{\hat{A}_{c i}}+1}$ if $\rho \lambda_{\tilde{A}_{c i}}+1 \neq 0$, or infinite poles if $\rho \lambda_{\tilde{A}_{c i}}+1=$ 0 .

4. The CRPD control law for $\hat{\Sigma}$ of $(1)$ is $u(t)=\tilde{K}(\rho \dot{x}(t)-$ $x(t))+w(t)$ where $\hat{K}=\tilde{K} T$.

It is well knowm Verghese (1981) that there are two classes of infinite poles: dynamic infinite poles and non-dynamic infinite poles. The impulsive responses are caused by dynamic infinite poles only. The effect of non-dynamic infinite poles is equivalent to direct response from input to state. For standard generalized system, the dynamic infinite poles are associated to the chained eigenvalues $\lambda_{A}$ of $A$, s.t. $\rho \lambda_{A}+1=0$; the non-dynamic infinite poles are associated to the non-chained $\lambda_{A}$, s.t. $\rho \lambda_{A}+1=0$. To avoid impulsive response in the closed-loop system, we should not select $\tilde{K}$ s.t. $\tilde{A}_{c} \triangleq \tilde{A}-\tilde{B} \tilde{K}$ has the chained $\lambda_{A}$, s.t. $\rho \lambda_{A}+1=$ 0.

\section{Numerical Example}

Consider the controllable continuous-time generalized systems of (1), where

$$
\begin{aligned}
\hat{E} & =\left[\begin{array}{llllll}
1 & 0 & 0 & 0 & 0 & 0 \\
0 & 1 & 0 & 0 & 0 & 0 \\
0 & 0 & 1 & 0 & 0 & 0 \\
0 & 0 & 0 & 0 & 1 & 0 \\
0 & 0 & 0 & 0 & 0 & 0 \\
1 & 0 & 0 & 0 & 0 & 0
\end{array}\right], \\
\hat{A} & =\left[\begin{array}{llllll}
0 & 0 & 1 & 0 & 0 & 0 \\
1 & 0 & 0 & 0 & 0 & 0 \\
0 & 1 & 0 & 1 & 0 & 0 \\
0 & 0 & 0 & 1 & 0 & 0 \\
0 & 0 & 0 & 0 & 1 & 0 \\
1 & 0 & 0 & 0 & 0 & 1
\end{array}\right], \hat{B}=\left[\begin{array}{ll}
1 & 0 \\
0 & 0 \\
0 & 0 \\
0 & 0 \\
1 & 1 \\
0 & 0
\end{array}\right] .
\end{aligned}
$$

We shall find the CRPD feedback control law $u(t)=\tilde{K}(\rho \dot{x}(t)$ $x(t))+w(t)$, s.t. the close-loop poles are $-2,-2,-2,-2,-2$ and $\infty$ 
Solution:

We take $\rho=0.5$ so that $|\hat{E}-\rho \hat{A}| \neq 0$. The general form triple $\{\hat{E}, \hat{A}, \hat{B}\}$ is left multiplied by $(\hat{E}-\rho \hat{A})^{-1}$ and transformed to the standard form triple $\{E, A, B\}$ using (6), as follows:

$E=\left[\begin{array}{rrrrrr}1.1429 & 0.2857 & 0.5714 & 0.0000 & -0.5714 & 0.0000 \\ 0.5714 & 1.1429 & 0.2857 & 0.0000 & -0.2857 & 0.0000 \\ 0.2857 & 0.5714 & 1.1429 & 0.0000 & -1.1429 & 0.0000 \\ 0.0000 & 0.0000 & 0.0000 & 0.0000 & -2.0000 & 0.0000 \\ 0.0000 & 0.0000 & 0.0000 & 0.0000 & 0.0000 & 0.0000 \\ -0.8517 & 0.2857 & 0.5714 & 0.0000 & -0.5714 & 0.0000\end{array}\right]$,
$A=\left[\begin{array}{rrrrrr}0.2857 & 0.5714 & 1.1429 & 0.0000 & -1.1429 & 0.0000 \\ 1.1429 & 0.2857 & 0.5714 & 0.0000 & -0.5714 & 0.0000 \\ 0.5714 & 1.1429 & 0.2857 & 0.0000 & -2.2857 & 0.0000 \\ 0.0000 & 0.0000 & 0.0000 & -2.0000 & -4.0000 & 0.0000 \\ 0.0000 & 0.0000 & 0.0000 & 0.0000 & -2.0000 & 0.0000 \\ -1.7143 & 0.5714 & 1.1429 & 0.0000 & -1.1429 & -2.0000\end{array}\right]$,
and

$$
B=\left[\begin{array}{rr}
0.0000 & -1.1429 \\
0.0000 & -0.5714 \\
-2.0000 & -2.2857 \\
-4.0000 & -4.0000 \\
-2.0000 & -2.0000 \\
0.0000 & -1.1429
\end{array}\right]
$$

The controllability indices of $A$ are $\kappa_{1}=2$ and $\kappa_{2}=4$. The matrix $M$ using (10.a), is found as

$$
\begin{aligned}
& M=\left[\begin{array}{rrrrrr}
0.0000 & 0.0000 & -1.1429 & -0.9796 & -2.7988 & -5.3844 \\
0.0000 & 0.0000 & -0.5714 & -1.6327 & -2.3790 & -5.4910 \\
-2.0000 & 4.0000 & -2.2857 & 2.6122 & -10.822 & 10.8755 \\
-4.0000 & 16.0000 & -4.0000 & 16.0000 & -48.000 & 128.000 \\
-2.0000 & 4.0000 & -2.0000 & 4.0000 & -8.0000 & 16.0000 \\
0.0000 & 0.0000 & -1.1429 & 3.5918 & -8.0233 & 16.2599
\end{array}\right], \\
& \text { and the matrix } T(10 . c) \text { is } \\
& T=\left[\begin{array}{rrrrrr}
2.2500 & -3.8750 & 1.7500 & 0.1250 & -2.0000 & -0.7500 \\
-1.5000 & 1.7500 & 0.0000 & -0.2500 & 0.0000 & 1.5000 \\
-0.2734 & 0.6562 & -0.4375 & 0.0000 & 0.4375 & 0.0547 \\
0.3281 & -0.4375 & 0.0000 & 0.0000 & 0.0000 & -0.1094 \\
-0.2188 & 0.0000 & 0.0000 & 0.0000 & 0.0000 & 0.2187 \\
-0.4375 & 0.0000 & 0.0000 & 0.0000 & 0.0000 & -0.4375
\end{array}\right]
\end{aligned}
$$

The compute CRPD controllable canonical form $\{\tilde{E}, \tilde{A}, \tilde{B}\}$ using (11), is

$$
\begin{aligned}
& \tilde{A}=T A T^{-1}
\end{aligned}
$$

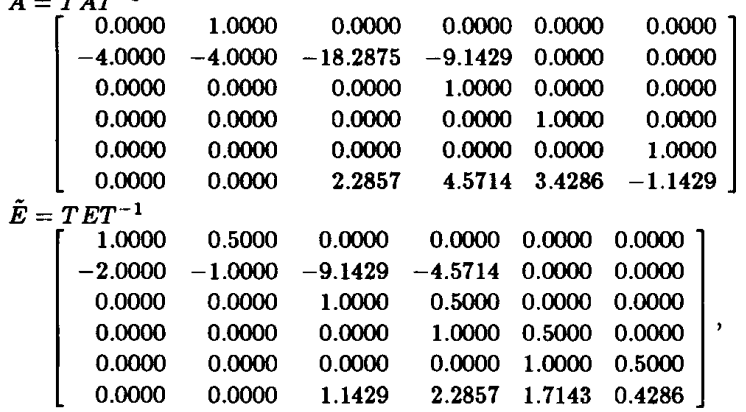

$$
\begin{aligned}
\tilde{B} & =T B \\
& =\left[\begin{array}{ll}
0.0000 & 0.0000 \\
1.0000 & 0.0000 \\
0.0000 & 0.0000 \\
0.0000 & 0.0000 \\
0.0000 & 0.0000 \\
0.0000 & 1.0000
\end{array}\right] .
\end{aligned}
$$

By Theorem 1, the desired closed-loop finite poles $\lambda_{R_{c i}}=-2$ are associated to the eigenvalues of $\tilde{A}_{c}, \lambda_{\tilde{A}_{c i}}=-1$ since $\lambda_{R_{c i}}=$
$\frac{\lambda_{\dot{A}_{c i}}}{\rho \lambda_{\lambda_{e i}}+1}$. Also, the desired closed-loop infinite pole is associated to the eigenvalues of $\tilde{A}_{c}, \lambda_{\bar{A}_{c}}=-2$ since $\rho \lambda_{\bar{A}_{c}}+1=0$. So, we shall find the state-feedback gain matrix $\tilde{K}$ such that the eigenvalues of are $-1,-1,-1,-1,-1$ and -2 . One possible $\tilde{A}_{c}$ is

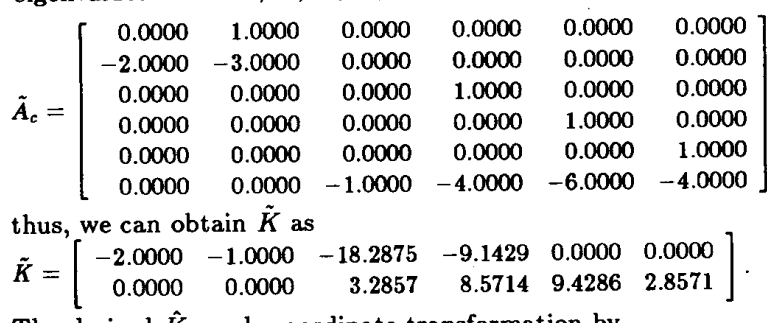
The desired $\hat{K}$ needs coordinate transformation by $\hat{K} \triangleq \tilde{K} T$

$$
\left[\begin{array}{rrrrrr}
-1.0000 & -2.0000 & 4.5000 & 0.0000 & -4.0000 & 0.0000 \\
-1.3984 & -1.5937 & -1.4375 & 0.0000 & 1.4375 & 0.0547
\end{array}\right] \text {. }
$$

To check the result, we apply the control law $u(t)=\hat{K}(\rho \dot{x}(t)-$ $x(t))+w(t)$ to the general form system $\hat{E} \dot{x}(t)=\hat{A} x(t)+\hat{B} u(t)$. We have

$$
\begin{aligned}
& \hat{A}_{c}=\hat{A}-\hat{B} \hat{K} \\
& {\left[\begin{array}{rrrrrr}
1.0000 & 2.0000 & -3.5000 & 0.0000 & 4.0000 & 0.0000 \\
1.0000 & 0.0000 & 0.0000 & 0.0000 & 0.0000 & 0.0000 \\
0.0000 & 1.0000 & 0.0000 & 1.0000 & 0.0000 & 0.0000
\end{array}\right]} \\
& \left.\begin{array}{llllll}
1.0000 & 0.0000 & 0.0000 & 0.0000 & 0.0000 & 0.0000 \\
0.0000 & 1.0000 & 0.0000 & 1.0000 & 0.0000 & 0.0000 \\
0.0000 & 0.0000 & 0.000 & 1.000 & 0.000 & 0.0000
\end{array}\right]
\end{aligned}
$$

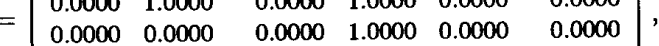

$$
\begin{aligned}
& \begin{array}{lllllll}
2.3984 & 3.5938 & -3.0625 & 0.0000 & 3.5625 & -0.0547
\end{array} \\
& \left.\begin{array}{llllll}
1.0000 & 0.0000 & 0.0000 & 0.0000 & 0.0000 & 1.0000
\end{array}\right] \\
& \hat{E}_{c}=\hat{E}-\rho \hat{B} \hat{K} \\
& =\left[\begin{array}{rrrrrr}
1.5000 & 1.0000 & -2.2500 & 0.0000 & 2.0000 & 0.0000 \\
0.0000 & 1.0000 & 0.0000 & 0.0000 & 0.0000 & 0.0000 \\
0.0000 & 0.0000 & 1.0000 & 0.0000 & 0.0000 & 0.0000 \\
0.0000 & 0.0000 & 0.0000 & 0.0000 & 1.0000 & 0.0000 \\
1.1992 & 1.7969 & -1.5313 & 0.0000 & 1.2813 & -0.0273 \\
1.0000 & 0.0000 & 0.0000 & 0.0000 & 0.0000 & 0.0000
\end{array}\right] \text {. }
\end{aligned}
$$

It can be computed that eigenvalues of $s \hat{E}_{c}-\hat{A}_{c}$ are $-2,-2,-2,-2$, -2 and $\infty$. So, the closed-loop eigenvalues are what we desired.

\section{Conclusion}

This paper proposes a conceptually and computationally simple method for assigning eigenvalues via CRPD feedback to a generalized system. The development depends crucially on properties of the standard generalized system, $E \dot{x}(t)=A x(t)+$ $B u(t)$, in which $\rho E-A=I$, such as

1. If $\lambda_{A}$ is an eigenvalue of $A$, and $\rho \lambda_{A}+1 \neq 0$, then $\lambda_{R}=$ $\frac{\lambda_{A}}{\rho \lambda_{A}+1}$ is an eigenvalue of $s E-A$.

2. If $\lambda_{A}$ is an eigenvalue of $A$ and $\rho \lambda_{A}+1=0$, then infinite is an eigenvalue of $s E-A$.

3. The standard generalized system is controllable if and only if

$$
\mathcal{C}=\left[B, A B, A^{2} B, \ldots, A^{n-1} B\right]
$$

has full rank.

4. The CRPD controllable canocical form are related to the controllable canonical form of $\{A, B\}$ pair.

The method developed allows us to use existing software packages (e.g. MATLAB, MATRIX ${ }_{X}$ ) to compute the solution. 


\section{Acknowledgment}

This work was supported in part by the National Science Council under Grand NSC 80-0404-E002-52.

\section{References}

[1] D. Cobb (1984). Controllability, observability, and duality in singular systems, IEEE Trans. Autom. Control, AC-29, 12, 1076-1082.

[2] C. T. Chen (1984). Linear System Theory and Design. New York, 345-346.

[3] F. R. Gantmacher (1974). The Theory of Matrices - volume two. Chelsea, New York, 25.

[4] F. L. Lewis (1986). A survey of linear singular systems. Circuits Systems Signal Process, 5, 1, 3-36.

[5] R. Mukundan and W. Dayawansa, (1983). Feedback control of singular systems - Proportional and derivative feedback of the state. Int. J. Systems Sci., 14, 6, 615-632.

[6] R. Nikoukhah, A. S. Willsky and B. C. Levy, (1987). Boundary-value descriptor systems: well-posedness, reachability and observability. Int. J. Control, 46, 5, 1715-1737.

[7] M. A. Shayman and Z. Zhou, (1987). Feedback control and classification of generalized singular systems. IEEE Trans. Autom. Control, AC-32, 6, 483-496.

[8] M. A. Shayman and Z. Zhou, (1987). On the control of generalized linear systems using constant-ratio proportional and derivative feedback. IFAC 10th triennial world congress, 127-132.

[9] V. L. Syrmos and F. L. Lewis, (1991). A geometric approach to proportional plus derivative feedback using quotient and partioned subspaces. Atomatica, 27, 2, 349-369.

[10] G. C. Verghese, B. C. Levy and T. Kailath, (1981). A generalized state space for singular systems. IEEE Trans. Autom. Control, AC-26, 4, 811-831.

[11] E. L. Yip and R. F. Sincovec, (1981). Solvability, controllability, and observability of continuous descriptor systems. IEEE Trans. Autom. Control, AC-26, 3, 702-707.

[12] Z. Zhou (1984). Feedback synthesis of singular systems - a geometric approach. Ph.D. dissertation, Washington University.

(13) Z. Zhou, M. A. Shayman and T. Z. Tarn, (1987). Singular systems: A new approach in the time domain. IEEE Trans. Autom. Control, AC-32, 1, 42-50. 